Wolf-Rayet Phenomena in Massive Stars and Starburst Galaxies

Proceedings IAU Symposium No. 193, (C)1999 IAU

K.A. van der Hucht, G. Koenigsberger \& P.R.J. Eenens, eds.

\title{
Role of starbursts in LINERs
}

\author{
JieHao Huang, Y. Chen, L. Ji, and Q.S. Gu \\ Astronomy Department, Nanjing University, Nanjing, 210093, China
}

\begin{abstract}
We report our preliminary results on the role of starbursts in LINERs by use of Wolf-Rayet galaxies as a tool. The essence of this approach lies in the different FIR-radio relation of WR galaxies from the pure AGN's.
\end{abstract}

The energetics of LINERs has been a controversial issue since their definition by Heckman (1980). In recent years, several LINERs have been observed by HST, or large ground-base facilities, and unambiguous evidence has been found for their energetics (see, e.g., Ho 1999; Maoz et al. 1998 and references therein). Ho favours the point of the low-luminosity end of the AGN phenomena for the majority of LINERs, while Maoz et al. argue for nuclear starbursts on the other hand (see, also Heckman, these Proceedings). We have found that the different FIR-radio relation of Wolf-Rayet galaxies from the pure AGN's could serve as a tool to classify the energetics of LINERs, a tight FIR-radio correlation for known WR galaxies implies that both FIR and radio emission for these sources are controlled by on-going star-formation activities.

Indeed, LINERs probably shock-excited (NGC 404, NGC 4569, NGC 4736, NGC 5055, NGC 5194, NGC 7217, Arp 220, F12112+0305, F14308-1447, see Maoz et al. 1998; Ho 1999; Ptak et al. 1998; Larkin et al. 1998; Taniguchi et al. 1999) basically illustrate a same FIR-radio correlation as WR galaxies, with lower radio emission in general (see Fig. 1a), and LINERs whose energy sources are composed of AGN and nuclear starbursts (NGC 4594, NGC 6240, UGC 5101, see Ptak et al. 1998; Schulz et al. 1999; Genzel et al. 1997) follow the same FIR-radio correlation as WR galaxies, with higher radio emission (see Fig. 1a). Interestingly, LINERs powered by AGN (M 87, NGC 1052, NGC 3998, NGC 4203, NGC 4278, see Ho 1999; Ptak et al. 1998; Iyomoto et al. 1998; Falcke et al. 1999) show a same FIR-radio relation as the majority of Sy1 galaxies, distinct from the FIR-radio correlation for WR galaxies (see Fig. 1b). M 81 is a source that Ho claims to be an AGN (Ho 1999), however, Maoz et al. thought that the contribution from massive stars could not be excluded (Maoz et al. 1998). According to our analysis, M 81 is a composite object, consistent with the results obtained from ROSAT data (Colbert \& Mushotzky 1999). NGC 6500 was considered as a composite object (Barth et al. 1997), though it is located definitely in the AGN regime in Fig. 1, in accordance with the new results by Falcke et al. (1998). Several kinds of predictions could be given based on this method, e.g., some of Sy galaxies may be powered by central black holes and their nuclear starbursts, as well as classification of LINERs' energetics, and further study of the approach we proposed here is underway. 


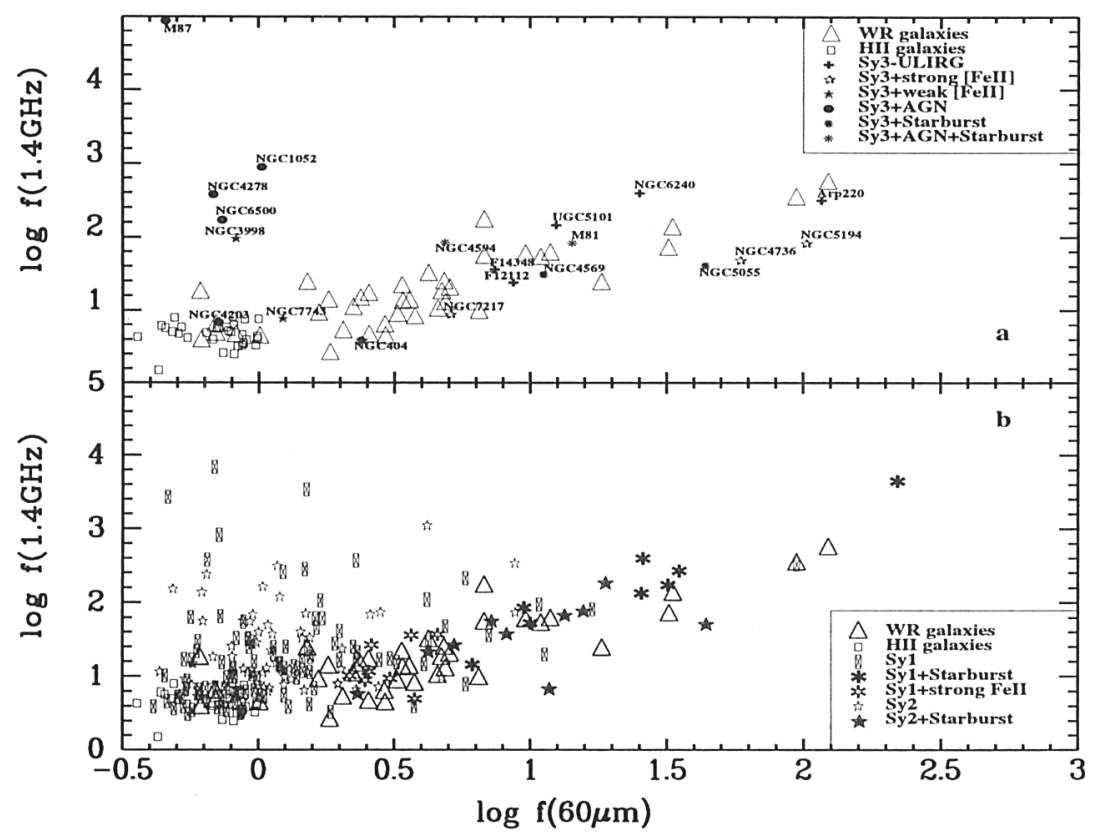

Figure 1. FIR-radio relation of WR galaxies, compared with (a) AGN- or starburst-dominated, or composite LINERs; (b) pure or composite AGNs.

\section{References}

Barth, A.J., Reichert, G.A., Ho, L.C. et al. 1997, AJ 114, 2313

Colbert, E.J.M., Mushotzky, R.F. 1999, in: Proc. 32nd COSPAR Meeting, AGNNormal Galaxy Connection, in press

Falcke, H., Ho, L.C., Ulvestad, J.S., et al. 1999, in Proc. Symp. on Astrophysics Research and Science Education (Vatican Obs.), in press

Genzel, R., Lutz, D., Sturm, E. et al. 1998, ApJ 498, 579

Heckman, T.M. 1980, A\&A 87, 152

Ho, L.C. 1999, in: Proc. 32nd COSPAR Meeting, AGN-Normal Galaxy Connection, in press

Iyomoto, N., Makishima, K., Matsushita, K. et al. 1998, ApJ 503, 168

Larkin, I.E., Armus, L., Knop, R.A., Soifer, B.T., Matthews, K. 1998, ApJS 114, 59

Maoz, D., Koratkar, A.P., Shields, J.C. et al. 1998, AJ 116, 55

Ptak, A., Yaqoob, T., Mushotzky, R., Serlemitsos, P., Griffiths, R. 1998, ApJ 501, L37

Schulz, H., Komossa, S., Greiner, J. 1999, in: Proc. Highlights in X-ray Astronomy, in press

Taniguchi, Y., Ohyama, Y. 1998, ApJ 508, L13 